\title{
Editor's Note: Forthcoming 30th Anniversary of Applied Intelligence
}

Published online: 17 June 2020

(C) Springer Science+Business Media, LLC, part of Springer Nature 2020
Applied Intelligence (APIN) has published 29 years of cuttingedge scholarly research and will be celebrating 30 years in 2021 with a selection of papers to commemorate this event. As we approach this anniversary, we now look back at the highlights over nearly three decades.

With a 2018 Impact Factor of 2.882, Applied Intelligence ranks in the second quartile for Artificial Intelligence in the JCR and has a steadily rising Impact Factor. The Web of Science identifies 21 notable, highly cited articles, and we expect that number to grow in the next decade.

Over nearly thirty years, Applied Intelligence has published more than 2000 articles in artificial intelligence and related areas, which have been indexed and downloaded widely. The journal is now published monthly with over 200 articles per year.

We look forward to many more years publishing research on the relevant flagship topics, including the following:

- Machine learning as it relates to classification and learning, emerging new technologies on deep learning, and neural networks then applying them to security systems, machine vision, economic forecasts, and healthcare.

- Optimization of algorithms for an array of applications, by employing evolutionary techniques, swarm intelligence, multi-objective systems, and nonlinear systems.
- New trends in granular computing and intelligent decision systems, including automatic decision support systems and recommender systems.

- Human-computer interaction and cognitive system relative to brain-system interface, cyber-physical system, natural language interfaces, and evolving systems.

- Big data analytics and data driven information systems, data, pattern, text, and rule mining, and knowledge representation and acquisition.

- Uncertain systems, computational intelligence dealing with uncertainty, and employing new concepts in emerging theories and practices.

- Intelligent systems' applications related to various domains including pattern recognition, image processing, intelligent robotic systems, searching, reasoning, diagnosis, and planning.

Publisher's note Springer Nature remains neutral with regard to jurisdictional claims in published maps and institutional affiliations. 